Supporting Information

\title{
Surface Modification and Functionalization of Boron Nitride Nanotubes via Condensation with Saturated and Unsaturated Alcohols for High Performance Polymer Composites
}

Kamia K. Smith ${ }^{a *}$, Neil D. Redeker ${ }^{b}$, Juan C. Rios ${ }^{b}$, Matthew H. Mecklenburgc, Jacob C. Marcischak $^{\mathrm{a}}$, Andrew J. Guenthner ${ }^{\mathrm{a}, \mathrm{d}}$, Kamran B. Ghiassi ${ }^{\mathrm{a} *}$

a Air Force Research Laboratory, Aerospace Systems Directorate, Edwards AFB, CA 93524

b ERC, Incorporated, Air Force Research Laboratory, Edwards AFB, CA 93524

${ }^{\mathrm{c}}$ Core Center of Excellence in Nano Imaging, University of Southern California, Los Angeles, CA 90089

d Nano Hydrophobics Inc., Lawrence Berkeley National Laboratory, Berkeley, CA 94720

E-mail: kamia.smith.2@us.af.mil,kamran.ghiassi@us.af.mil

\section{Table of Contents}

1. FTIR

2. NMR

3. SEM 


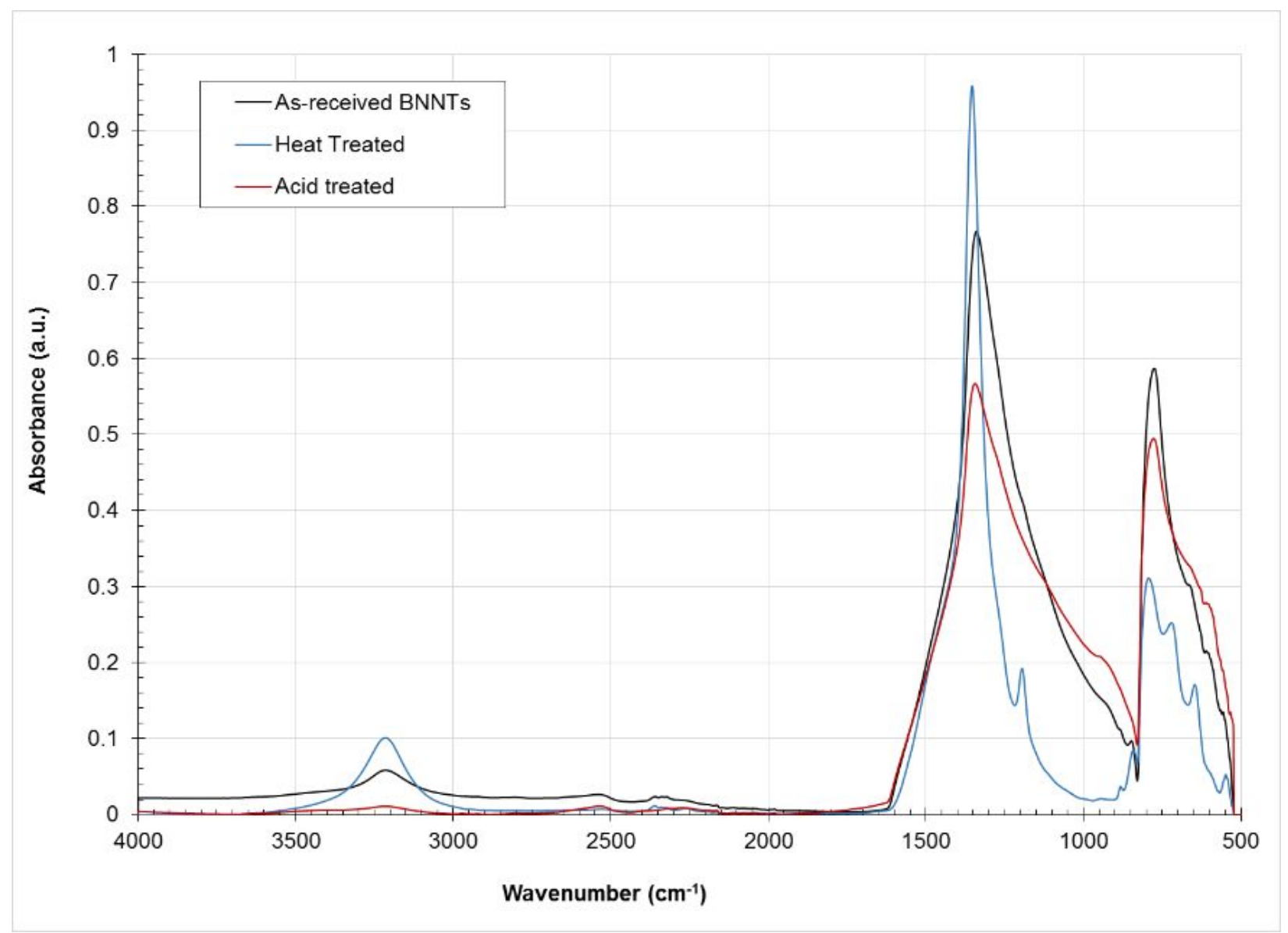

Figure S1. FTIR-ATR spectra of as-received BNNTs, heat-treated BNNTs, and acid-treated BNNTs. The broad peak at $3250 \mathrm{~cm}^{-1}$ for the as-received and heat-treated BNNTs is characteristic of the $\mathrm{B}_{2} \mathrm{O}_{3}$ vibrations. Heat-treated BNNTs (before methanol wash) show increased levels of oxidation on the B-N in-plane peak as well as the B-N-B bending peak. Spectra were recorded at 32 scans and $8 \mathrm{~cm}^{-1}$ resolution. 


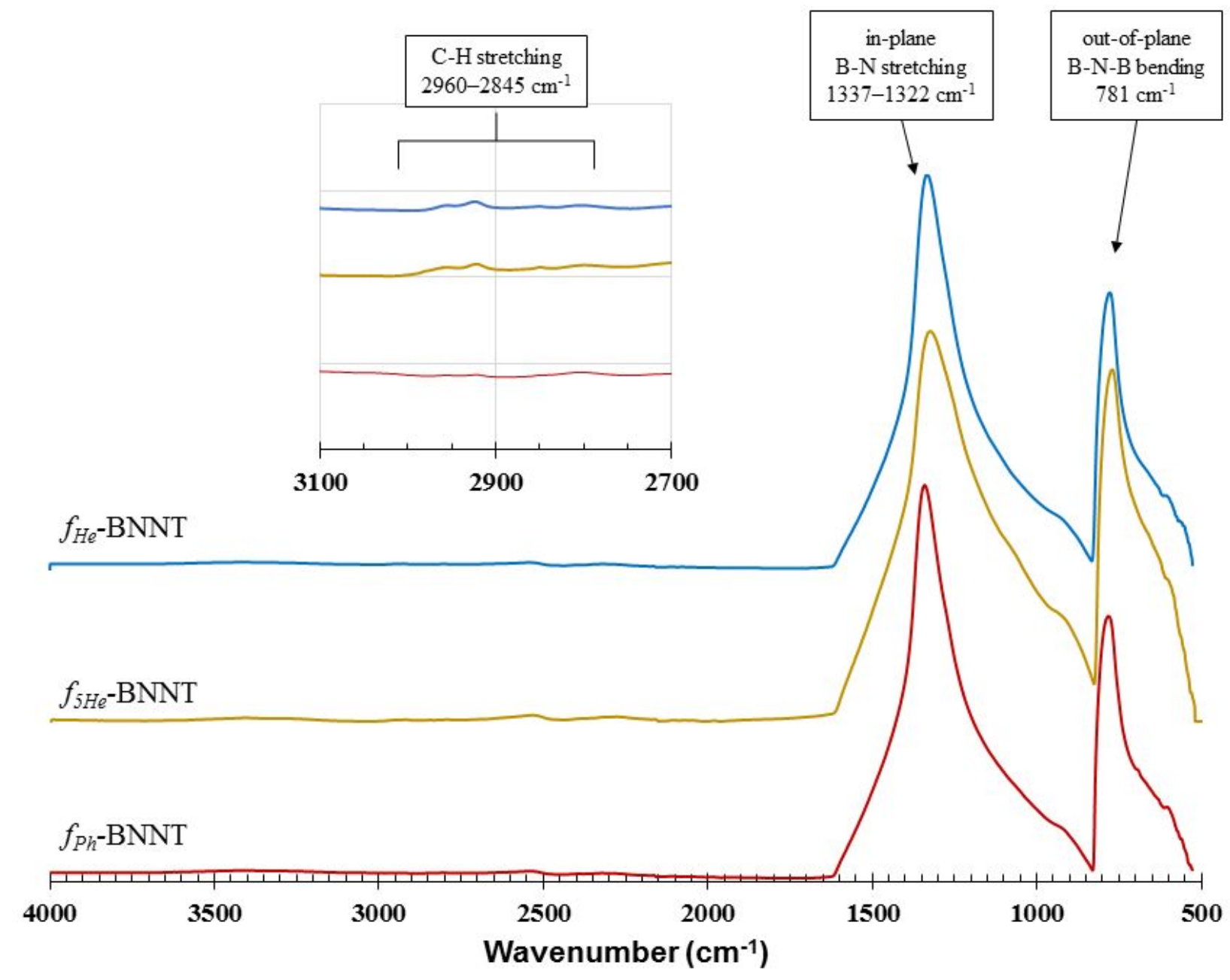

Figure S2. FTIR-ATR spectra of the remaining three compounds from this investigation: $f_{\mathrm{He}^{-}}$ BNNT, $f_{5 H e}$-BNNT, and $f_{P h}$-BNNT. $f_{P r}$-BNNT is not included for reasons discussed in the main text. 


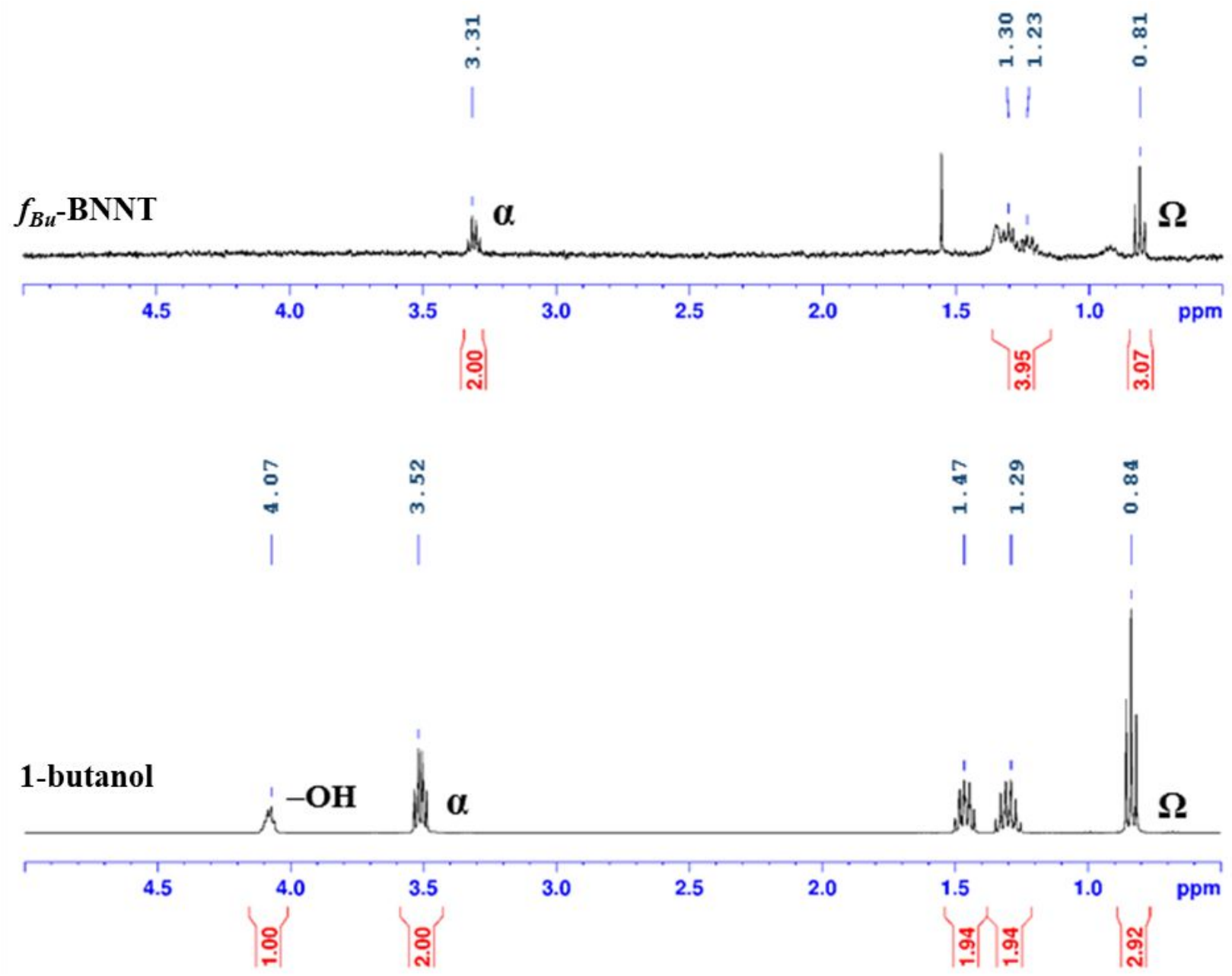

Figure S3. NMR spectra of $f_{B u}$-BNNT (top) compared to pristine 1-butanol (bottom) in benzene$d_{6}$. 


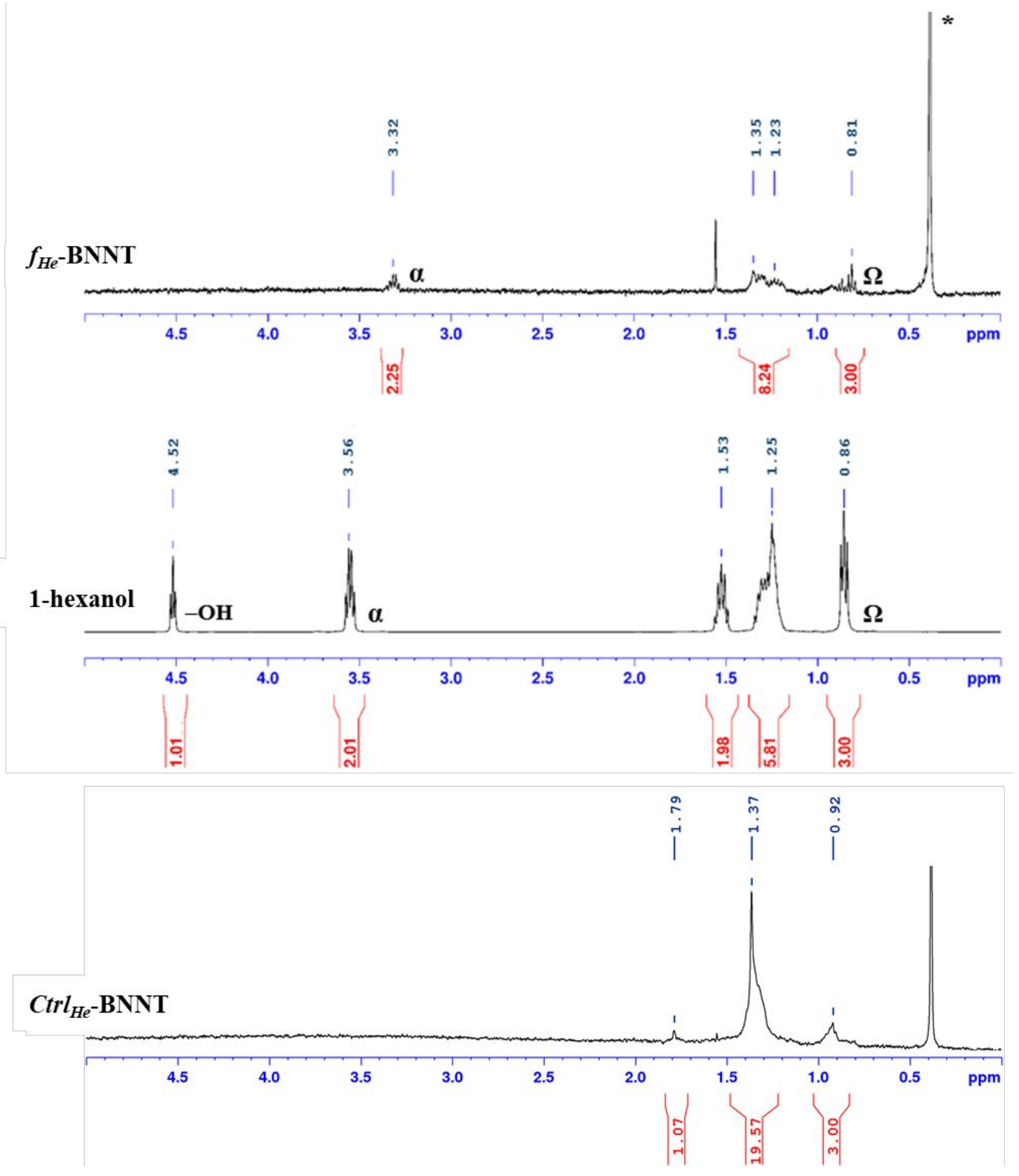

Figure S4. NMR spectra of $f_{\mathrm{He}}$-BNNT (top) compared to pristine 1-hexanol (middle) and $\mathrm{Ctrl}_{\mathrm{He}^{-}}$ BNNT (bottom) in benzene- $d_{6}$. Notably, in the control sample the $\alpha$-hydroxyl proton signals are absent, and the alkyl integrations are significantly changed from both the pristine 1-hexanol and functionalized samples, suggesting that the signals are not from covalently bonded or adsorbed 1hexanol. 


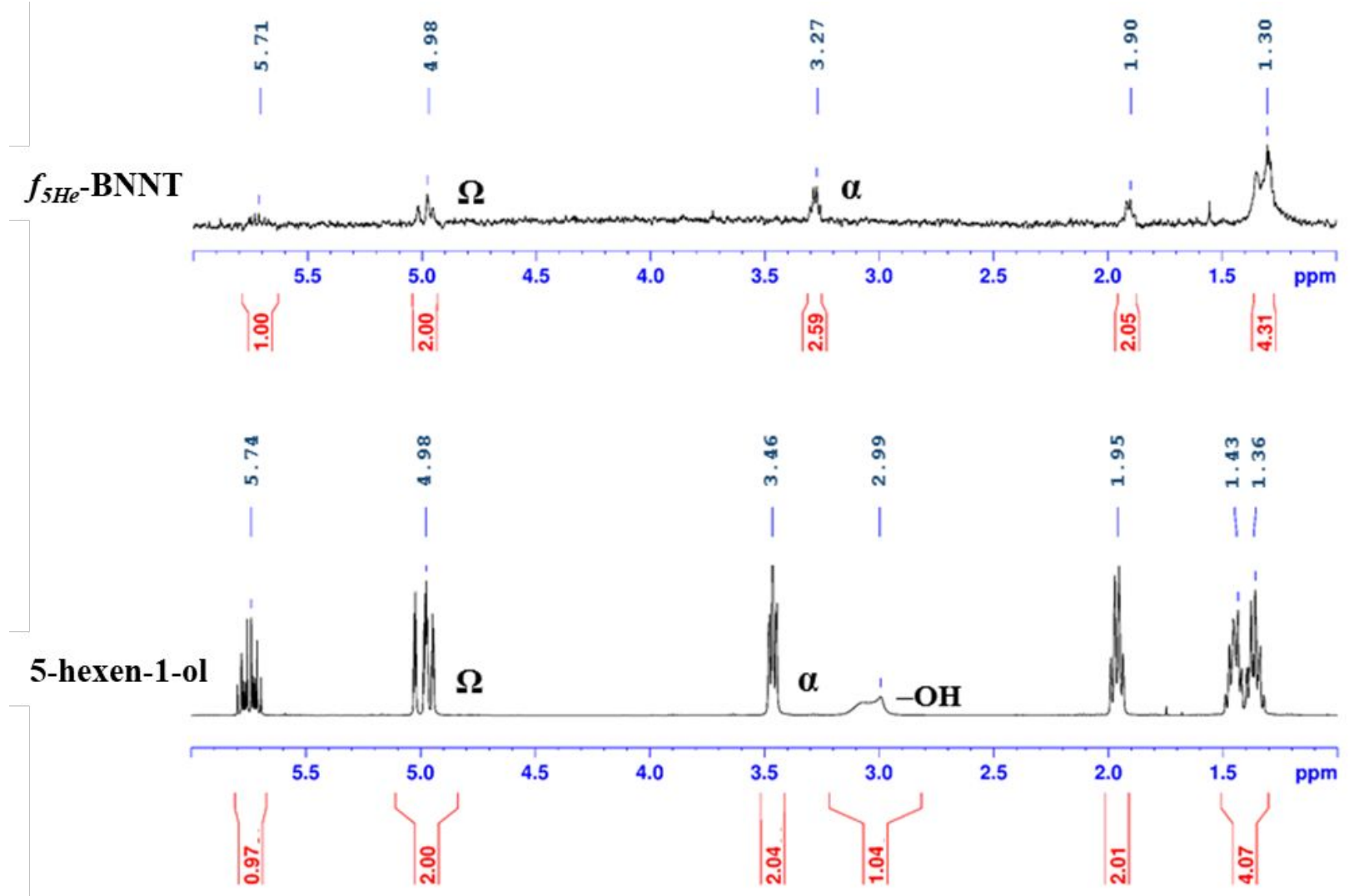

Figure S5. NMR spectra of $f_{5 H e}$-BNNT (top) compared to pristine 5-hexen-1-ol (bottom) in benzene- $d_{6}$. 


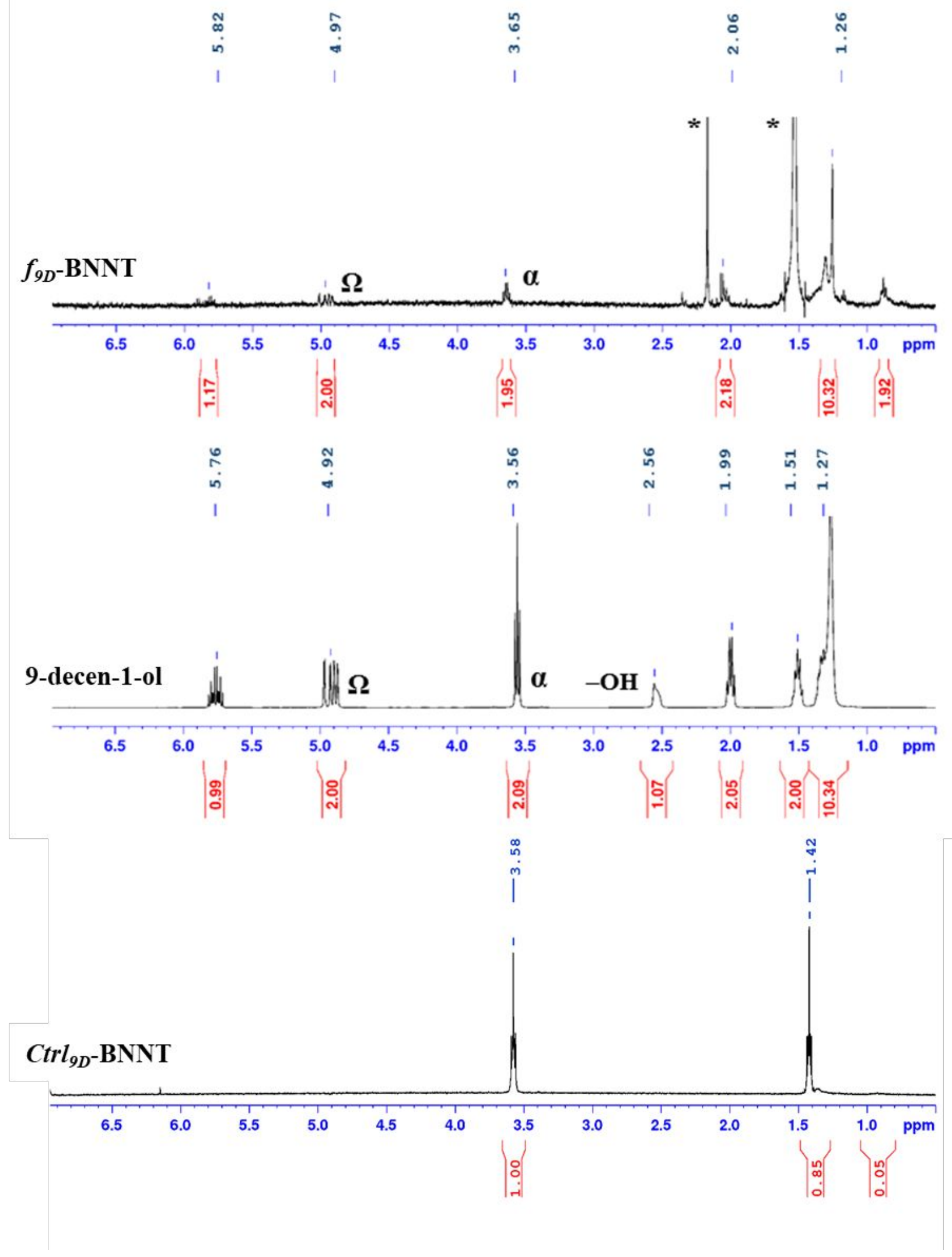


Figure S6. NMR spectra of $f_{9 D}$-BNNT (top) compared to pristine 9-decen-1-ol (middle) and $C_{t r} l_{9 D^{-}}$BNNT in benzene- $d_{6}$. Notably, the olefinic and alkyl proton signals are absent in the $C_{t r l} l_{9 D^{-}}$ BNNT sample compared to both pristine 9-decen-1-ol and the functionalized sample, suggesting that the signals are not due to covalently bonded or adsorbed 9-decene-1-ol.

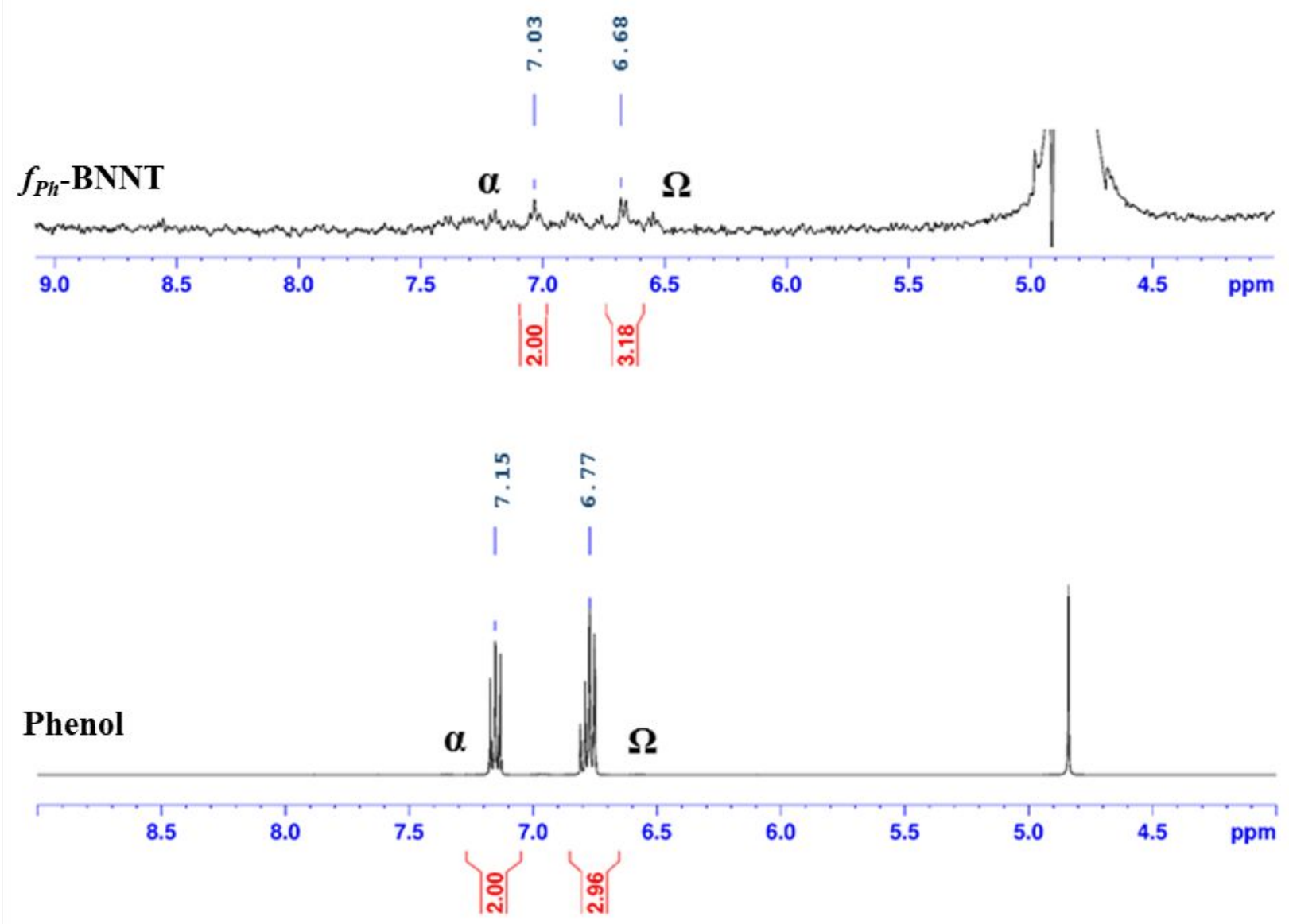

Figure S7. NMR spectra of $f_{P h}$-BNNT (top) compared to pristine phenol (bottom) in methanol$d_{4}$. Note that, in the case of phenol, the phenolic proton is obfuscated by the water peak, which occurs in methanol- $d_{4}$ at $4.85 \mathrm{ppm}$. This is confirmed in Figure S-8. 


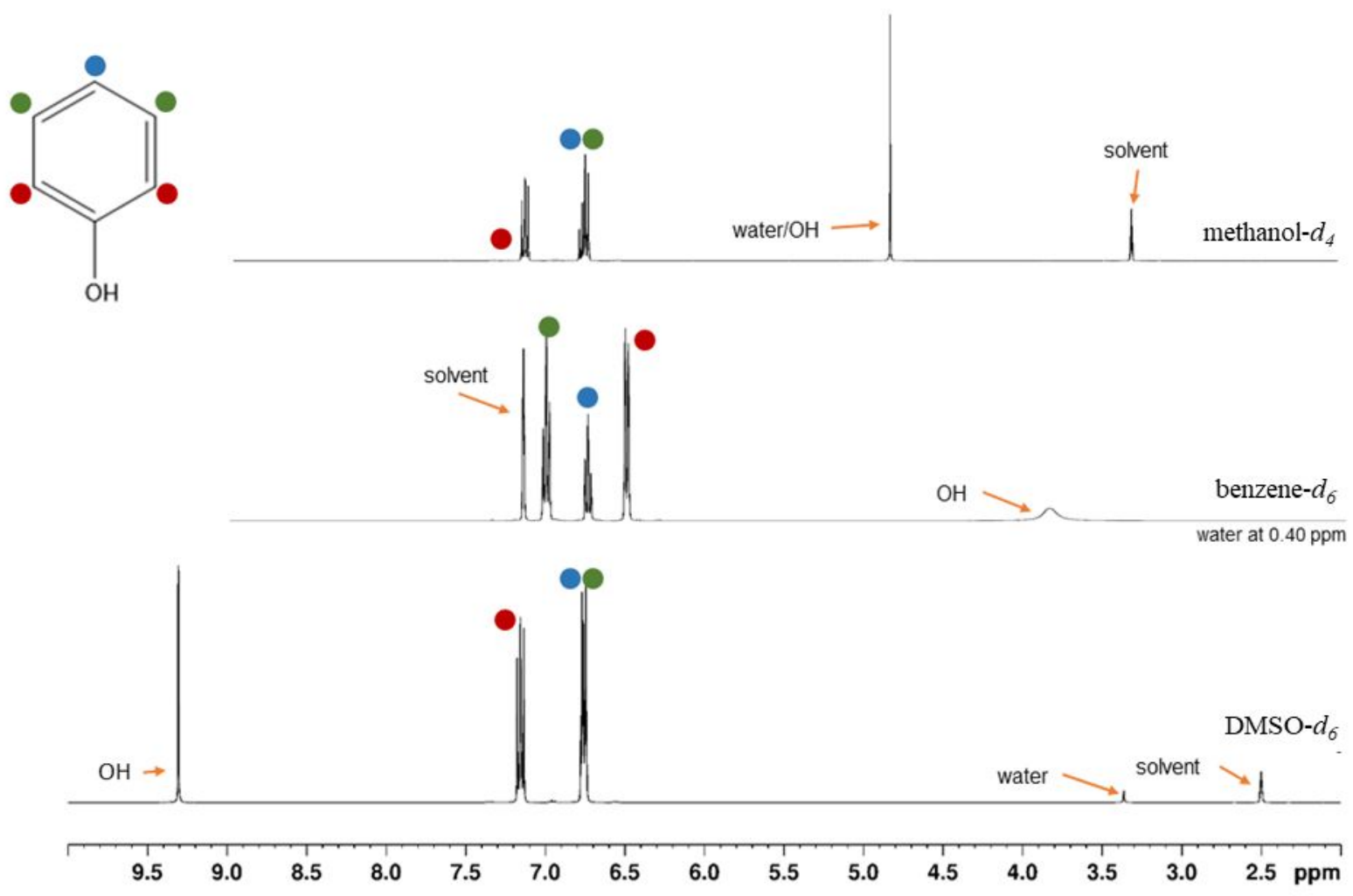

Figure S8. Comparison of pure phenol in three different NMR solvents, methanol- $d_{4}$ (top), benzene- $d_{6}$ (middle), and DMSO- $d_{6}$ (bottom). The red, green, and blue circles denote the ortho, $m e t a$, and para proton positions on the phenol ring. For methanol- $d_{4}$, the water and $\mathrm{OH}$ peak are overlapping. For benzene- $d_{6}$, the $\mathrm{OH}$ is seen as a broad peak around $3.86 \mathrm{ppm}$, and the water peak comes at $0.40 \mathrm{ppm}$ but is not shown in this spectra. For DMSO- $d_{6}$, the $\mathrm{OH}$ peak appears at 9.31 ppm. 

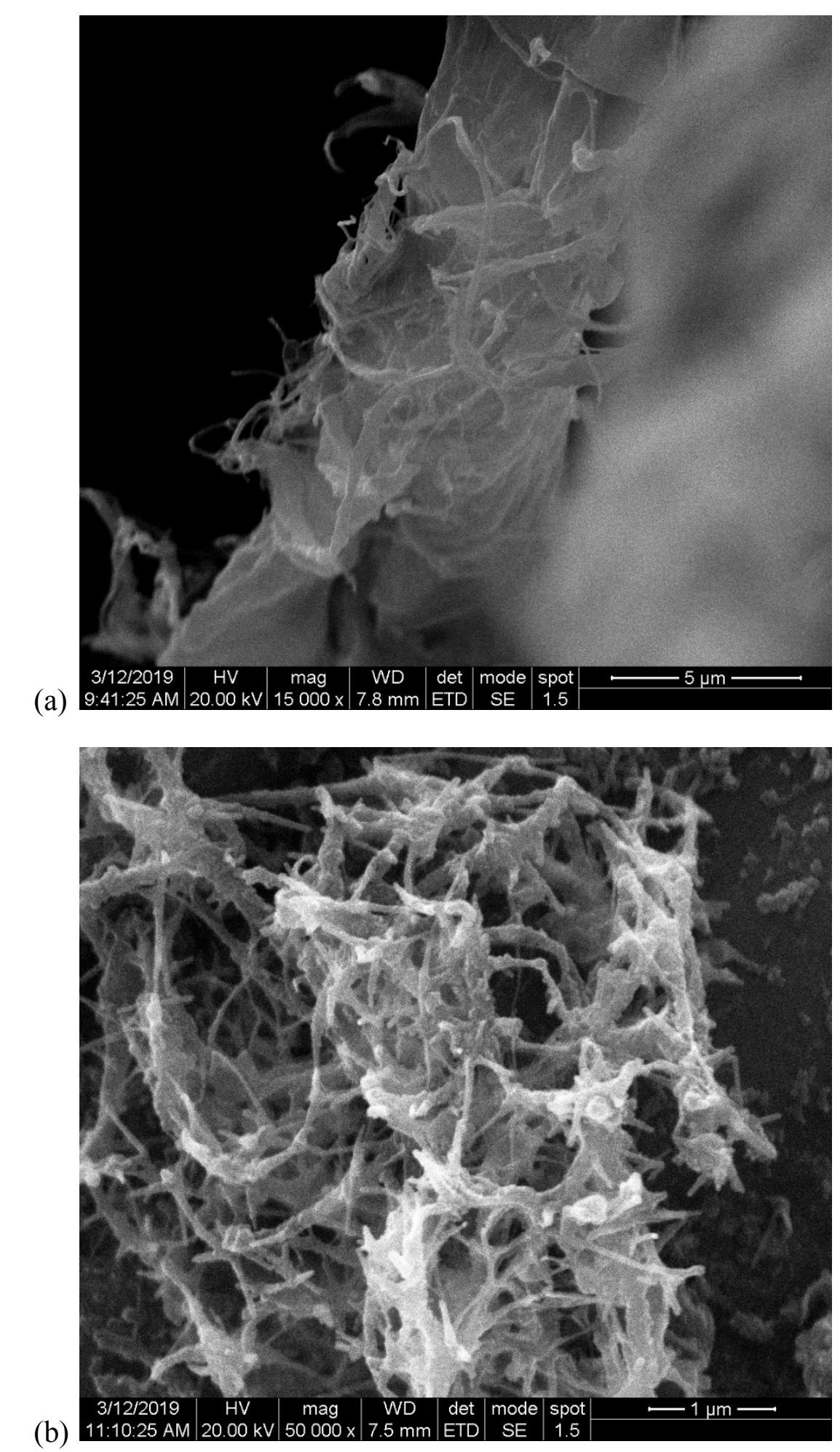

Figure S9. SEM micrographs of (a) as-received nanotubes and (b) immediately after acid treatment. Lengths range from 1-20 $\mu \mathrm{m}$. 


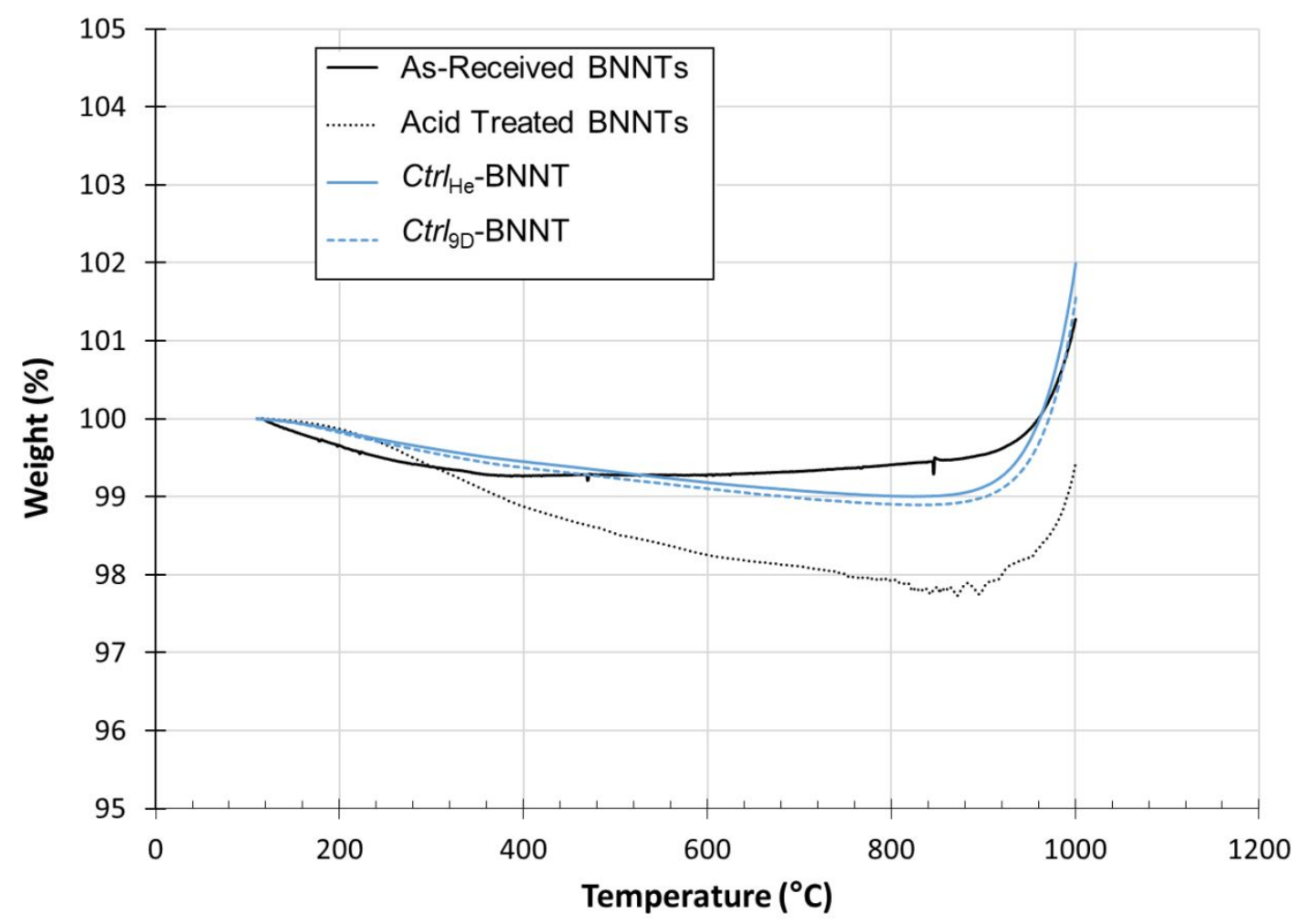

Figure S10. Thermogravimetric analysis plots of BNNT control samples including as-received, after acid treatment, and as-received samples which were stirred in 1-hexanol or 9-decene-1-ol. Control samples prepared using non-acid treated BNNTs show oxidation temperature and initial weight loss comparable to as-received samples. 